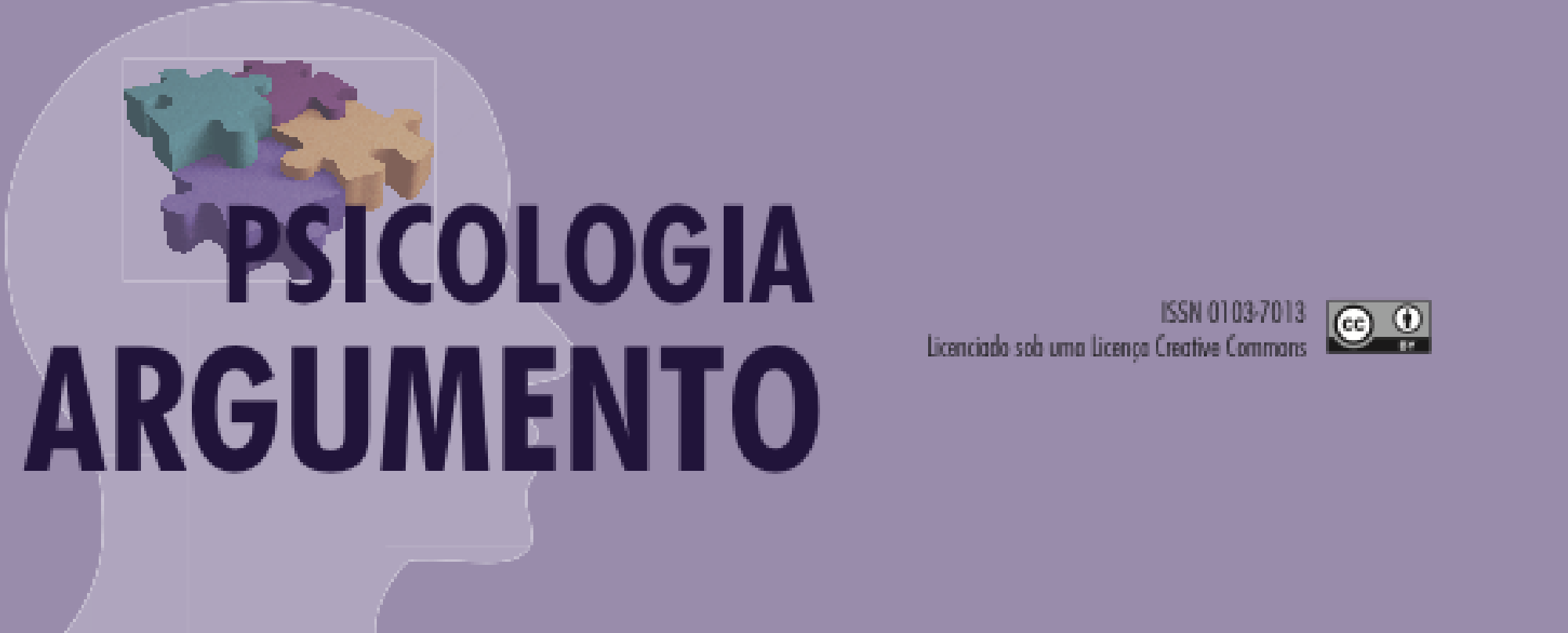

doi: http://dx.doi.org/10.7213/psicolargum.37.97.A006

\title{
Ideação suicida e psicopatologia em amostra brasileira de tatuados e não tatuados
}

Suicidal ideation and psychopathology in a brazilian sample of tattooed and nontattooed

Ideación suicida y psicopatología en una muestra brasileña tatuada y no tatuada

\section{Adriano Schlösser}

Universidade do Oeste de Santa Catarina/Campus Videira,Email: adriano.s@unoesc.edu.br. Orcid: https://orcid.org/0000-0002-1728-1414

\author{
Brigido Vizeu Camargo \\ Universidade Federal de Santa Catarina. Orcid: https://orcid.org/0000-0002-9529-4923 \\ Andréia Isabel Giacomozzi \\ Universidade Federal de Santa Catarina. Orcid: https://orcid.org/0000-0002-3172-5800
}

Mestranda Juliana Gomes Fiorott

Universidade Federal de Santa Catarina. Orcid: https://orcid.org/0000-0002-6064-1593

\section{Resumo}

O objetivo deste estudo foi investigar a saúde mental e psicopatologia associada entre indivíduos tatuados e não tatuados, de ambos os sexos. Participaram 614 indivíduos, divididos entre $306 \mathrm{com}$ tatuagem e $308 \mathrm{sem}$ tatuagem. Foi desenvolvido um questionário online auto administrado, com respostas abertas e fechadas. O questionário apresentou 2 blocos de informações: a) questões sociodemográficas: idade, estado civil, religião, escolaridade e quantidade de tatuagens; $b$ ) comportamento de risco: transtornos psicológicos e ideação suicida. Para análise de dados, foi 
realizada análise estatística descritiva e relacional, com auxílio do programa estatístico SPSSversão 17.0. Os resultados indicaram associações estatisticamente significativas associadas a diagnóstico psiquiátrico entre mulheres tatuadas, e ideação suicida entre mulheres não tatuadas. Embora com resultados estatisticamente significativos, ressalta-se que os valores apresentados entre tatuados e não tatuados foram muito semelhantes, indicando-se cautela em associar comportamentos desviantes a indivíduos com tatuagens.

Palavras chave: comportamento de risco; tatuagem; psicologia.

\begin{abstract}
To investigate the mental health and associated psychopathology among tattooed and nontattooed individuals of both sexes. Participated in the study 614 individuals, divided between 306 with tattoo and 308 without tattoo. A self-administered online questionnaire was developed, with open and closed answers. The questionnaire presented two blocks of information: a) sociodemographic issues: age, marital status, religion, educational level and number of tattoos; $b$ ) risk behavior: psychological disorders and suicidal ideation. For data analysis, a descriptive and relational statistical analysis was performed, using the statistical program SPSS - version 17.0. The results indicated statistically significant associations connected with psychiatric diagnosis among tattooed women. Although with statistically significant results, it is emphasized that the values presented between tattooed and not tattooed were very similar, indicating caution in associating psychiatric diagnosis with individuals with tattoos.
\end{abstract}

Keywords: risk behavior; tattoo; psychology.

\title{
Resumen
}

El objetivo de este estudio fue investigar la salud mental y la psicopatología asociada entre individuos tatuados y no tatuados de ambos sexos. Participaron 614 individuos, divididos entre 306 con tatuaje y 308 sin tatuaje. Se desarrolló un cuestionario en línea autoadministrado con respuestas abiertas y cerradas. El cuestionario presentó 2 bloques de información: a) preguntas sociodemográficas: edad, estado civil, religión, educación y número de tatuajes; b) conducta de riesgo: trastornos psicológicos e ideación suicida. Para el análisis de datos, se realizó un análisis estadístico descriptivo y relacional con la ayuda del programa estadístico SPSS-versión 17.0. Los resultados indicaron asociaciones estadísticamente significativas asociadas con el diagnóstico psiquiátrico entre las mujeres tatuadas y la ideación suicida entre las mujeres no tatuadas. Aunque con resultados estadisticamente significativos, es notable que los valores presentados entre tatuados y no tatuados fueron muy similares, lo que indica precaución al asociar el comportamiento desviado con individuos con tatuajes.

Palabras clave: comportamiento de riesgo; tatuaje Psicologia

\section{Introdução}

Operacionalmente, a tatuagem é um procedimento realizado no corpo, que imprime uma marca permanente através de um desenho sob a superfície da pele (Durkin, 2012; King \& Vidourek, 2013; Kluger, 2014; Stirn, Oddo, Peregrinova, Philipp, \&Hinz, 2011;Valentí, 2009). Ela é realizada por meio de um dispositivo, que injeta um pigmento 
ou corante permanente através de punções na derme, podendo apenas ser retirada por cirurgia ou laser (Tiggemann \& Hpkings, 2011).

A tatuagem constitui-se como uma prática cada vez mais presente na sociedade e por isso tem se tornado alvo de estudos científicos, em diversas áreas do conhecimento, como no campo antropológico, sociológico, criminológico e em diversas áreas da saúde (Abonizio, 2011; Armstrong, DeBoer, \& Cetta, 2008; Bicca, Duquia, Breunig, Souza, \& Almeida, 2013; Braithwaite, Robillard, Woodring, Stephen, \& Arriola, 2001; Brooks, Woods, Knight, \& Shrier, 2003; Caroni, \& Grossman, 2012; Ferreira, 2011; 2010; 2008; 2007; Osório, 2006; Pérez, 2006; Sabino, \& Luz, 2006; Satchithananda, Walsh, \& Schofield, 2001; Shebani, Miles, Simmons, \& De Giovanni, 2007; Vail, 1999). Devido a relevância do tema em sua dimensão psicológica e psiquiátrica, estudos nacionais e internacionais têm sido realizados sob diferentes perspectivas, visando compreender a analisar a relação da tatuagem com diversas esferas da saúde mental.

Nesse sentido, estudos internacionais têm apresentado resultados sobre a relação entre tatuagem e distúrbios alimentares, depressão, comportamentos suicidas e suicídio, agressividade, baixa tolerância à frustração e impulsividade, dependência à tatuagem, comportamento criminoso, dentre outros (Braithwaite, Robillard, Woodring, Stephen, \& Arriola, 2001; Byard \& Charlwood, 2014; Brooks et al., 2003; Deschesnes, Finès, \& Demers, 2006; Heywood et al., 2012; Jennings, Fox, \& Farrington, 2014; King \& Vidourek, 2013; Koch, Roberts, Armstrong, \& Owen, 2010; Krasic, Mitic, Kostic, Ilic, \& Rankovic, 2011; Solano et al, 2014; Stirn, Oddo, Peregrinova, Philipp, \& Hinz, 2011; Preti et al., 2006; Sosin, 2014; Stirn et al., 2012; Yen et al, 2012).

A partir do exposto, o presente estudo objetivou investigar a saúde mental e psicopatologia associada entre indivíduos tatuados e não tatuados, de ambos os sexos a fim de verificar se tais associações encontradas em outros países também se apresentam no contexto brasileiro.

\section{Método}

O estudo possui natureza descritiva e comparativa, com desenho transversal (Marconi \& Lakatos, 2007). Participaram 614 indivíduos, divididos entre 306 com 
tatuagem e 308 sem tatuagem. A Tabela 1 apresenta a distribuição de frequência dos participantes.

\section{Tabela 1.}

Distribuição dos participantes considerando sexo e grupo.

\begin{tabular}{|c|c|c|c|c|c|c|}
\hline \multirow[t]{2}{*}{ Sexo } & \multicolumn{3}{|c|}{ Grupos } & \multirow[b]{2}{*}{$\%$} & \multirow[b]{2}{*}{ Total } & \multirow[b]{2}{*}{$\%$} \\
\hline & Tatuados & $\%$ & Não tatuados & & & \\
\hline Masculino & 83 & 27,1 & 90 & 29,2 & 173 & 28,2 \\
\hline Feminino & 223 & 72,9 & 218 & 70,8 & 441 & 71,8 \\
\hline Total & 306 & 100 & 308 & 100 & 614 & 100 \\
\hline
\end{tabular}

Devido à diferença na quantidade de participantes entre os sexos, para análises intersexo, foi feito uso de seleção randômica, contabilizando 80 participantes por sexo e presença ou não de tatuagem, totalizando 160 participantes para esta modalidade de análise. As análises intrasexo foram realizadas com os valores totais dos participantes, tendo por critério de inclusão a participação de indivíduos com idade acima de 18 anos.

\section{Instrumento}

Foi desenvolvido um questionário online auto administrado com respostas abertas e fechadas. O questionário foi composto por: a) questões sócio demográficas: idade, estado civil, escolaridade e quantidade de tatuagens; b) transtornos psicológicos e ideação suicida.

\section{Procedimento de coleta de dados}

Realizou-se contato por meio de e-mail, estúdios de tatuagem,redes sociais e contato pessoal, de acordo com o critério de inclusão. Foi feito uso como técnica suplementar a bola de neve, onde participantes poderiam indicar aos conhecidos responderem o questionário. Um estudo piloto foi realizado previamente com 20 participantes, visando verificar se o questionário respondia aos objetivos da pesquisa.

\section{Análise de dados}

Realizou-se análise estatística descritiva (média, desvio padrão e distribuição de frequências) e relacional (teste do Qui-quadrado e teste-t de Student). Os dados foram 
digitados em uma planilha e analisados com o auxílio do programa estatístico Statistical Package for Social Sciences (SPSS- versão 17.0).

\section{Aspectos éticos}

O projeto obteve parecer favorável do Comitê de Ética em Pesquisa com Seres Humanos da UFSC, sob protocolo n. 1.353.995, e todas as diretrizes éticas foram seguidas, seguindo as normas da Resolução n. 510/2016 do Conselho Nacional de Saúde sobre pesquisas envolvendo seres humanos nas Ciências Humanas e Sociais. Foi solicitada aos participantes a leitura e aceite do Termo de Consentimento Livre e Esclarecido, presente na primeira etapa do questionário.

\section{Resultados}

A média geral de idade dos participantes do sexo masculino foi 30,9 $(D P=10,24)$, enquanto do sexo feminino foi 30,3 $(D P=10,1)$. Para o grupo de tatuados do sexo masculino, a média de idade foi 29,2 ( $D P=6,57)$, e a de não tatuados foi de 32,7 ( $D P=$ 12,48). Para o sexo feminino, a média de idade entre as participantes com tatuagem foi de 29,06 $(D P=8,17)$, e 31,7 $(D P=11,56)$ para não tatuadas.

Dos 173 homens, 39,3\% afirmaram estarem casados ou em um relacionamento estável, seguidos de 37,6\% solteiros, 20,8\% namorando e 2,3\% divorciados. Das 441 mulheres, 38,3\% declararam-se casadas ou em um relacionamento estável, 31,2\% solteiras, $23,8 \%$ namorando, $6,3 \%$ divorciadas e $0,4 \%$ viúvas. Não houve diferença estatisticamente significativa entre ter tatuagem e estado civil.

Com relação à escolaridade, verificou-se que a maioria dos participantes $(53,6 \%)$ possui Ensino Superior - completo ou incompleto, seguido de pós-graduação - completa ou incompleta. Ressalta-se que os valores são diferenciados entre os sexos devido à diferença da amostra. A análise da amostragem randômica não identificou associação estatisticamente significativa entre ter tatuagem e grau de escolaridade.

Sobre saúde mental, 19,9\% do total da amostra afirmou ter algum diagnóstico psiquiátrico. Contudo, não foi identificada associação estatisticamente significativa nas análises intergrupo e intersexo. A Tabela 2 apresenta os dados referentes à esta variável. 
Tabela 2.

Diagnóstico psiquiátrico e frequência, de acordo com sexo e grupo dos participantes.

\begin{tabular}{|c|c|c|c|c|c|}
\hline \multirow[t]{2}{*}{ Diagnóstico } & \multicolumn{2}{|l|}{ Masculino } & \multicolumn{2}{|l|}{ Feminino } & \multirow[t]{2}{*}{ Total } \\
\hline & Tatuados & Não tatuados & Tatuadas & Não tatuadas & \\
\hline $\begin{array}{l}\text { Transtorno } \\
\text { Depressivo }\end{array}$ & 04 & 14 & 34 & 10 & 62 \\
\hline $\begin{array}{l}\text { Transtorno de } \\
\text { Ansiedade }\end{array}$ & 02 & 04 & 10 & 05 & 21 \\
\hline $\begin{array}{l}\text { Transtorno do } \\
\text { Pânico }\end{array}$ & 02 & 01 & 04 & 07 & 14 \\
\hline $\begin{array}{l}\text { Transtorno } \\
\text { Bipolar }\end{array}$ & 01 & 00 & 04 & 00 & 05 \\
\hline Distúrbio do sono & 00 & 01 & 04 & 00 & 05 \\
\hline TOC & 01 & 02 & 01 & 01 & 05 \\
\hline $\begin{array}{l}\text { Transtornos de } \\
\text { Personalidade }\end{array}$ & 02 & 00 & 01 & 00 & 03 \\
\hline Distimia & 00 & 01 & 01 & 01 & 03 \\
\hline TDAH & 00 & 00 & 02 & 00 & 02 \\
\hline TEPT & 00 & 00 & 02 & 00 & 02 \\
\hline Total & 12 & 23 & 63 & 24 & 122 \\
\hline
\end{tabular}

Relacionando a variável "diagnóstico psiquiátrico" com "tatuagem”, observou-se que dentre os participantes do sexo feminino que afirmaram ter um diagnóstico psiquiátrico, $70,7 \%$ tem tatuagem e $29,3 \%$ não tem, enquanto que dentre as que declararam não ter diagnóstico, $52,9 \%$ não possui tatuagem. Houve associação estatisticamente significativa entre as variáveis $[\chi 2=12,44 ; \mathrm{gl}=1 ; p=.000]$. Para o sexo masculino, verificou-se uma inversão, havendo predomínio de não tatuados com diagnóstico psiquiátrico $(65,4 \%)$ em comparação com tatuados $(34,6 \%)$, embora o percentual seja semelhante entre os grupos, ao alegar não possuir diagnóstico psiquiátrico ( $51 \%$ tatuados e $49 \%$ não tatuados). Não houve associação significativa entre as variáveis.

Sobre já ter pensado em suicídio, 34,3\% do total de participantes afirmaram já terem pensado. Destes, 65,6\% não possuem tatuagem e 34,4\% possuem, havendo associação estatisticamente significativa a partir da amostra randômica $[\chi 2=1,51 ; \mathrm{gl}=1$; 
$p<.05]$. Com relação aos sexos, dos participantes que afirmaram terem tido ideação suicida, a partir da amostra randômica 50,9\% são mulheres e 49,1\% são homens, não havendo associação estatisticamente significativa.

A maioria dos participantes do sexo masculino afirmou nunca ter pensado em suicídio (65,3\%), com associação estatisticamente significativa $[\chi 2=6,04 ; \mathrm{gl}=1 ; p<.05]$. No caso das mulheres, não houve associação estatisticamente significativa entre as variáveis tatuagem e ideação suicida $[\chi 2=0,016 ; \mathrm{gl}=1$; NS], visto que $17,7 \%$ das participantes sem tatuagem e 17,3\% das tatuadas afirmaram já ter pensado em suicídio.

\section{Discussão}

Ao longo de décadas, considerou-se que indivíduos tatuados são mais propensos ao uso de álcool, cigarro e outras substâncias psicoativas, comportamento violento, violações de trânsito, propensão à ter sido preso, criminalidade, impulsividade, dentre outros (Adams, 2009; Drews et al.,2000; Forbes, 2001; Gittleson \& Wallen, 1973; Greif, Hewitt, \& Armstrong, 1999; King \& Vidourek, 2013; Pozgain,Barkić, Filaković, \&Koić,2004; Zrno, Frencl, Degmecic, \& Pozgain, 2015), levando-a associarse à características negativas (Durkin \& Houghton, 2000). Contudo, as produções recentes têm apresentado dados divergentes. Estudos recentes indicam que indivíduos com modificações corporais não são mais susceptíveis de envolvimento em comportamentos de risco quando comparados com pessoas não modificadas (GilesGorniak, Vandehey, \& Stiles, 2016, Swami et al., 2015).

Com relação a saúde mental das participantes, não houve associação significativa entre os grupos de tatuados e não tatuados. Estudo recente proposto por Giles-Gorniak et al. (2016) indicou que indivíduos com tatuagem não são susceptíveis de se envolver em comportamentos de risco ou reportar um histórico de problemas de saúde mental quando comparados com pessoas sem tatuagem. Nas análises intragrupos, para o sexo feminino, das participantes que afirmaram possuir algum diagnóstico, a maioria era de tatuadas. Tatuagens têm sido associadas a psicopatologia e desvios (D’Ambrosio, Martini, \& Casillo, 2014; Hawkes, Senn, \& Thorn, 2004). O maior número de casos referindo-se a transtorno depressivo e/ou transtorno de ansiedade na amostra deste estudo 
também foi verificado em pesquisas anteriores (Nathanson, Paulhus, \& Williams, 2006; Roberti \& Storch, 2005; Yen et al., 2012; Zrno et al., 2015).

No entanto, o estudo de Fredrick e Bradley (2000) aponta que indivíduos tatuados apresentavam escores de depressão significativamente menores do que os participantes não tatuados, indo ao encontro dos resultados deste estudo no grupo masculino, com maior quantidade de não tatuados apresentando depressão. Concorda-se aqui com Stirn e Hinz (2008), de que as práticas de modificação corporal podem variar de imitações de grupos de pares a sintomas informativos de condições psicopatológicas possivelmente graves, sendo necessário cautela em atribuir problemas psicológicos em indivíduos com modificações corporais (Swami et al., 2016; Tate \& Shelton, 2008).

Também não foi identificado associação entre tatuagem e ideação suicida, sendo esta uma ligação sugerida em adultos (Solano et al., 2014; Yen et al., 2012), inclusive com maior associação para mulheres tatuadas (Carrol et al.,2002). Nossos resultados concordam com os resultados recentes de King e Vidourek (2013), não relacionando comportamento suicida à prática da tatuagem.

De modo geral, ressalta-se que a amostra, embora aleatória, teve um alto índice de participantes com ensino superior e pós-graduação, seja de tatuados como de não tatuados. Possivelmente poderiam ser identificadas práticas sociais, motivações e comportamentos de risco diferentes caso tivessem participado com maior proporção indivíduos com educação formal mais baixa. Não obstante, isso é apenas uma hipótese, sem evidências que sustentem esta conjectura, o que não invalida os resultados, mas apresenta-se como limitação de pesquisa, que pode ser realizada em outro contexto.

\section{Considerações finais}

Os resultados do presente estudo apontaram associações estatisticamente significativas associadas a diagnóstico psiquiátrico entre mulheres tatuadas, e ideação suicida entre mulheres não tatuadas. Embora com resultados estatisticamente significativos, ressalta-se que os valores apresentados entre tatuados e não tatuados foram muito semelhantes, indicando-se cautela em associar comportamentos desviantes a indivíduos com tatuagens. 


\section{Referências}

Adams, J. (2009). Marked difference: tattooing and its association with deviance in the United States. Deviant Behavior, 30, 266-292. doi: https://doi.org/10.1080/01639620802168817.

Carroll, S.T., Riffenburgh, R.H., Roberts, T.A., \& Myhre, E.B. (2002). Tattoos and body piercings as indicators of adolescent risk-taking behaviors. Pediatrics, 109, 10211027. doi: http://dx.doi.org/10.1542/peds.109.6.1021.

D'Ambrosio, A., Martini, V., \& Casillo, N. (2014). Piercings and tattoos: Psychopathological aspects. Giornale di Neuropsichiatria dell'Età Evolutiva, 34(1), 1-10.

Drews, D. R., Allison, C. K., \& Probst, J. R. (2000). Behavioral and self-concept differences in tattooed and nontattooed college students. Psychological Reports, 86 , 475-481. doi: http://dx.doi.org/10.2466/pr0.2000.86.2.475.

Durkin, K., \& Houghton, S. (2000). Children's and adolescents' stereotypes of tattooed people as delinquent. Legal and Criminological Psychology, 5, 153-164. doi: https://doi.org/10.1348/135532500168065.

Forbes, G. B. (2001). College students with tattoos and piercings: Motives, family experiences, personality factors, and perception by others. Psychological Reports, 89, 774-786. doi: https://doi.org/10.2466/pr0.2001.89.3.774.

Fredrick, C. M., \& Bradley, K. A. (2000). A different kind of normal? Psychological and motivational characteristics of young adult tattooers and body piercers. North American Journal of Psychology, 2, 379-389.

Giles-Gorniak, A. N., Vandehey, M. A., \& Stiles, B. L. (2016). Understanding Differences in Mental Health History and Behavioral Choices in a Community Sample of Individuals with and without Body Modifications. Deviant Behavior, 37(8), 852860. doi: https://doi.org/10.1080/01639625.2015.1060798.

Gittleson, L., \& Walleng, D. I. (1973) The tattooed male patient. British Journal of Psychiatry,122, 295-300. doi: https://doi.org/10.1192/bjp.122.3.295.

Greif,J., Hewitt,W., \& Armstrong, M. L.(1999).Tattooing and body piercing: Bodyart practices among college students. Clinical Nursing Research, 8, 368-385. doi: https://doi.org/10.1177/10547739922158368.

Hawkes, D., Senn, C. Y., \& Thorn, C. (2004). Factors that influence attitudes toward women with tattoos. Sex Roles, 50, 593-604. doi: https://doi.org/10.1023/B:SERS.0000027564.83353.06. 
King, K. A., \& Vidourek, R. A. (2013). Getting inked: Tattoo and risky behavioral involvement among university students. The Social Science Journal, 50(4), 540-546. doi: https://doi.org/10.1016/j.soscij.2013.09.009.

Nathanson, C., Paulhus, D.L., \& Williams, K.M. (2006). Personality and misconduct correlates of body modification and other cultural deviance markers. Journal of Research in Personality,40, 779-802. doi: https://doi.org/10.1016/j.jrp.2005.09.002.

Pozgain, I., Barkić, J., Filaković, P., \& Koić, O. (2004). Tattoo and personality traits in Croatian veterans. Yonsei Medical Journal, 45(2), 300-305. doi: https://doi.org/10.3349/ymj.2004.45.2.300.

Roberti, J. W., \& Storch, E. A. (2005). Psychosocial adjustment of college students with tattoos and piercings. Journal of College Counseling, 8, 14-19. doi: https://doi.org/10.1002/j.2161-1882.2005.tb00068.x.

Solano, P., Magagnoli, M., Pizzorno, E., Innamorati, M., Bruzzone, L., \& Amore, M. (2014). EPA-1070 - Suicide attempts and "body-art": is there any correlation? a preliminary study. European Psychiatry, 29(1), 1. doi: https://doi.org/10.1016/S09249338(14)78351-8.

Stirn, A., \& Hinz, A. (2008). Tattoos, body piercings, and self-injury: Is there a connection? Investigations on a core group of participants practicing body modification. Psychotherapy research, 18(3), 326- 333. doi: https://doi.org/10.1080/10503300701506938.

Swami, V., Gaughan, H., Tran, U. S., Kuhlmann, T., Stieger, S., \& Voracek, M. (2015). Are tattooed adults really more aggressive and rebellious than those without tattoos? Body Image, 15, 149-152. doi: https://doi.org/10.1016/j.bodyim.2015.09.001.

Swami, V., Tran, U. S., Kuhlmann, T., Stieger, S., Gaughan, H., \& Voracek (2016). More similar than different: Tattooed adults are only slightly more impulsive and willing to take risks than Non-tattooed adults. Personality and Individual Differences, 88, 4044. doi: https://doi.org/10.1016/j.paid.2015.08.054.

Tate, J. C., \& Shelton, B. L. (2008). Personality correlates of tattooing and body piercing in a college sample: The kids are alright. Personality and Individual Differences, 45, 281-285. doi: https://doi.org/10.1016/j.paid.2008.04.011.

Yen, C-F., Hsiao, R. C., Yen, J-Y., Yeh, Y-C., Wang, P-W., Lin, H-C., \& Ko, C-H. (2012). Tattooing among high school students in southern Taiwan: The prevalence, correlates and associations with risk-taking behaviors and depression. The Kaohsiung Journal of Medical Sciences, 28(7), 383-389. doi: https://doi.org/10.1016/j.kjms.2011.10.008. 
Zrno, M., Frencl, M., Degmecic, D., \& Pozgain, I. (2015). Emotional profile and risk behaviours among tattooed and non-tattooed students. Medicinski Glasnik, 12(1), 9398. 